Article

\title{
Interdisciplinary Alliances to Deploy Telemedicine Services in Isolated Communities: The Napo Project Case
}

\author{
Ignacio Prieto-Egido ${ }^{1,2, *(\mathbb{D})}$, Javier Simó-Reigadas ${ }^{1}$ and Andrés Martínez-Fernández ${ }^{1}$ \\ 1 Signal Theory and Communications Department, Rey Juan Carlos University, Camino Del Molino s/n, \\ Fuenlabrada, 28943 Madrid, Spain; javier.simo@urjc.es (J.S.-R.); andres.martinez@urjc.es (A.M.-F.) \\ 2 EHAS Foundation, ETSI de Telecomunicación, Ciudad Universitaria s/n, 28040 Madrid, Spain \\ * Correspondence: ignacio.prieto@urjc.es; Tel.: +34-914-888-741
}

Received: 18 June 2018; Accepted: 25 June 2018; Published: 2 July 2018

\begin{abstract}
Rural telecommunications projects in developing regions have a long history of unsuccessful experiences due to the complexity of such projects: Practically none of the dimensions of integral sustainability are straight forward in such projects. When sustainability becomes a priority, it is difficult for traditional alliances of development to account for all critical success factors. In the Peruvian Amazon Rainforest, some institutions have developed rural telecommunications projects along the Napo River during the last 10 years. The experience has shown at each stage what aspects of sustainability were not taken into account because the partners involved did not have all the needed capacities, and this has made the alliances of partners evolve in a sustainability-driven manner. This paper analyzes these cases and assesses how sustainability has evolved in relation to the structure of such alliances.
\end{abstract}

Keywords: rural telecommunications; integral sustainability; heterogeneous multi-partner alliances

\section{Introduction}

Prospective studies state that $70 \%$ of the world population will live in cities by 2050. Following this trend, the development of telecommunications technologies supporting new services is more and more focused on urban areas. Mobile cellular networks are a clear example: While 2G was deployed targeting full coverage of most of the territory in many countries, 3G was deployed with coverage concentrated in populated areas and $4 \mathrm{G}$ coverage maps showing smaller spots in the main cities.

Living conditions in isolated rural communities in developing regions are difficult because they often lack access to basic services such as electricity, water, sanitation, healthcare, and education. ICTs could be key enablers of access to some of those services, but the rural-urban digital divide gets larger and larger as new technologies assume the conditions of densely populated areas. The reason for the rural-urban digital divide is three-fold: a difficult rural geography, high poverty rates, and a highly sparse population. This leads to high infrastructure deployment costs and low expected revenues, a fatal combination that prevents telecommunication operators from investing in rural telecommunications infrastructures, at least using conventional technologies and business models.

In this context, the EHAS Foundation has been working on real rural telecommunications projects since 2004, aiming to find the optimal conditions for deploying sustainable rural communications networks in isolated areas of several countries in Latin America, and more intensively in Peruvian regions such as the mountainous areas in the Cuzco province and the Amazon rainforest in several parts of the Loreto region. During the last 14 years, the target of those rural telecommunications deployments has evolved from telemedicine networks to general purpose telecommunications infrastructures; technologies have evolved to enhance the robustness, the capacity, and the quality of service support; furthermore, the business 
models proposed have become more complex and better suited for long-term sustainability in those regions. As well, during that process, the partnerships and alliances have evolved as feedback from previous projects showed how sustainability runs into problems as it develops. a direct relationship between the structure of heterogeneous interdisciplinary partnerships and alliances and sustainability has become more and more obvious, evolving from basic yet complex three-party alliances among NGOs, universities, and public institutions, to larger, complex networks involving Mobile Network Operators (MNOs) and Small Rural Operators (SRO), and making clear what the role of each of them is for the success of the project and for long-term sustainability.

This paper illustrates and evaluates the evolution of partnerships and alliances explained above specifically for the case of the Napo Network, in the Peruvian Amazon rainforest, considering three stages: In stage 1 the alliances considered sufficient included universities, NGOs, and public governmental institutions; in stage 2, Mobile Network Operators (MNOs) and more diverse public institutions were added; in stage 3, Small Rural Operators (SRO) and other technological partners were also included in the consortium, and local organizations closer to communities where also included. a simple sustainability model is used to evaluate the relationship among those alliances and sustainability.

\section{State of the Art in Telecommunications Infrastructures and Services Sustainability}

Incumbent operators in developing countries are usually reluctant to deploy rural telecommunications infrastructures in sparsely populated areas far from cities. The case for mobile cellular networks clearly illustrates the dynamics of the market: a MNO (Mobile Network Operator) needs to invest a great deal of money and effort in order to reach many small villages over a large area, while the expected revenue is very low. Moreover, many governments leave MNOs free to decide whether they will start providing their services in small villages, but once they provide connectivity they are forced to stay; this is the case for Peru and explains why operators don't provide any connectivity at all over large remote rural areas.

Under those circumstances, many researchers and experts in ICT4D (Information and Communications Technologies for Development) are trying to find new paradigms for connecting the unconnected and for providing services to underserved communities. a variety of the different approaches is explained in a taxonomy of "alternative networks" proposed in [1]. In the first decade of the XXI century, some alternative telecommunications networks were pushed by technological groups that were sensitive to the reality of unconnected remote areas; these pilots showed, firstly, the technical difficulties of deploying low-cost infrastructures over regions that lack electricity, access to existing supporting structures for telecommunications equipment, technical staff for maintenance, etc. Clear examples are the first telemedicine networks promoted by our consortia in Latin America [2,3], experiences promoted by KTH in Africa [4], or networks deployed by the TIER group in Asia [5], among others. Some of the most persistent groups investigated how to make those pilots sustainable and went beyond the technical issues, proposing appropriate maintenance models that took into account the hazardous conditions of telecommunications infrastructures in remote, underserved rural areas; the TIER group [5] and the EHAS group [6] are only two examples among many others.

In a second stage, interdisciplinary groups focused on aspects such as management and real, long-term sustainability of rural telecommunication infrastructures in remote regions [7-9]. The relevance of the participation of local communities as fundamental actors in all the stages of ICT4D projects becomes more and more important $[4,7,10]$, and some research certainly points to the Community Networks paradigm [1] being the way to promote integrally sustainable and appropriate telecommunications solutions for rural communities. Relevant examples of this trend can be seen around Zenzeleni networks in South Africa [9], Rizhomatica in Mexico [11], and the bottom-up community cellular network in Papua presented in Reference [10].

However, from the "technical", top-down initiatives to more recent, bottom-up successful experiences, the big telecommunications operators have been seen as difficult partners from the point of view of all other stakeholders, as they are very powerful and have clear market-driven dynamics. The TUCAN3G 
project, funded by the European Commission, tried to fill this gap in the quest for solutions to connect the unconnected, and successfully deployed pilots of $3 \mathrm{G}$ cellular networks in remote areas involving an MNO [12,13]. TUCAN3G marked the guidelines for making alliances with different stakeholders that seek more sustainable and scalable rural telecommunications networks with the participation of an $\mathrm{MNO}$, an experience that should be seen as complementary to the also successful experiences of bottom-up initiatives with a strong emphasis on the role of communities.

\section{Methodology}

The raw material for this paper is the experience during a period of 10 years in the Napo Network, in which different projects have turned the initial telemedicine network deployed in 2008 along the Peruvian section of the Napo River into a combined infrastructure aiming at providing general telecommunications services in the region as well as improved telemedicine services.

The effectiveness of the alliances that developed this project at each stage for managing sustainability is partially based on the analytical framework proposed by References $[7,8]$. The same dimensions of integral sustainability will be used, but instead of going into the details of CSFs (Critical Success Factors) for a particular project, the impact of partners participating in alliances in each dimension of sustainability is going to be evaluated instead. an evaluation of the global contribution of each partner to each dimension of sustainability is done ex-post, deciding whether that partner contributes to it or not in the short-term and in the long-term, and to what extent.

Figure 1 shows the diagram that illustrates how the results of each stage in the Napo Project will be summarized in terms of sustainability management in Figures $2-4$. In that diagram, a connectivity icon is presented under the roles covered by the partners depending on the lever of contribution to the sustainability of the project. With this kind of graph, it is easy to show if a project is considering the different dimensions of sustainability and the different roles that are considered.

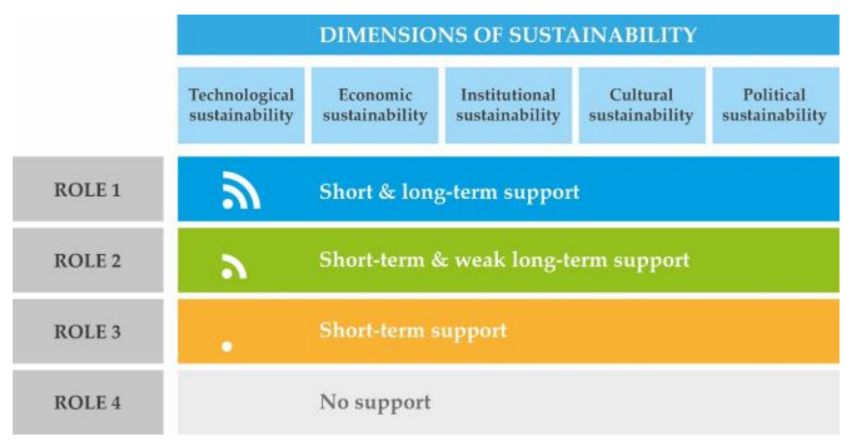

Figure 1. Graphic tool to represent differentiated contribution of partners to the accomplishment of each dimension of sustainability.

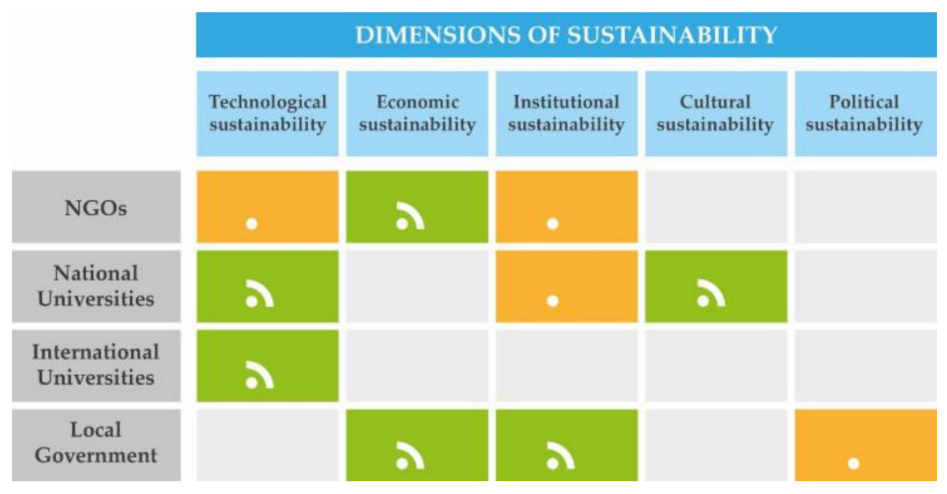

Figure 2. Sustainability analysis of the consortium for the first stage (color code: green for short-term and weak long-term support; orange for short-term support; and gray for no support). 


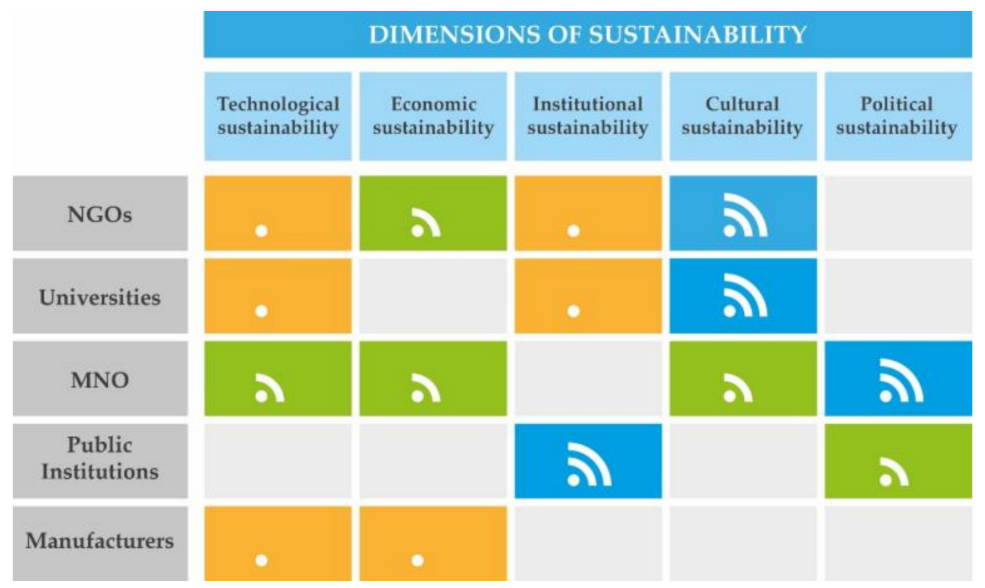

Figure 3. Sustainability analysis of the consortium for the second stage (color code: blue for short and long-term support; green for short-term and weak long-term support; orange for short-term support; and gray for no support).

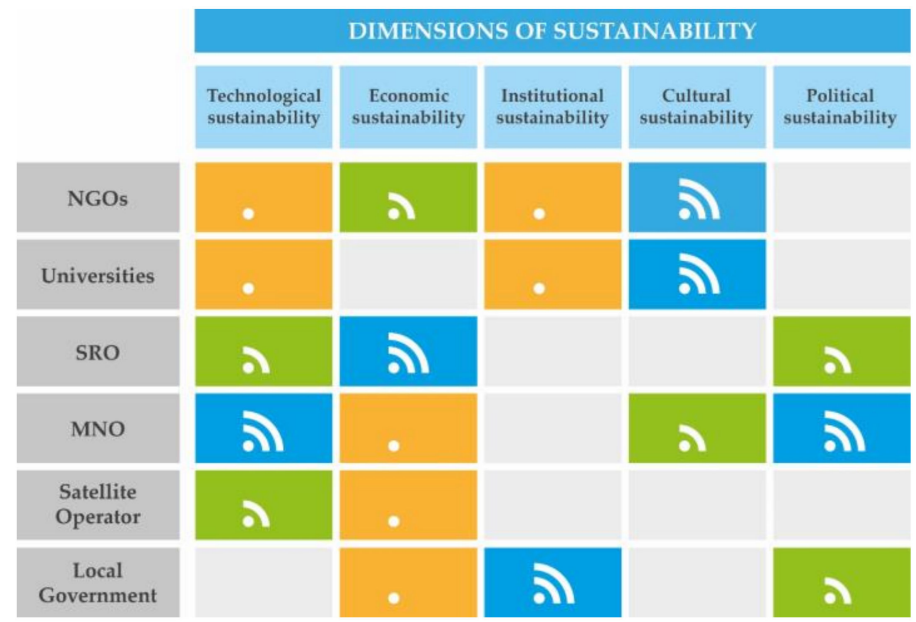

Figure 4. Sustainability analysis of the consortium for the third stage (color code: blue for short and long-term support; green for short-term and weak long-term support; orange for short-term support; and gray for no support).

\section{Deployment of Telemedicine Services Stage}

The Napo is an Amazonian river that begins in Ecuador and, after crossing to Peru, flows into the Amazon River. The section that passes through Peruvian territory, in which this article will focus, is about $450 \mathrm{~km}$ long and has more than 15 communities, most of them of indigenous population with less than 400 inhabitants. In this Amazon region there are very few roads and communications are usually made by river, which makes it slow and expensive to travel long distances due to the price of fuel. This difficulty to travel is combined with the lack of telecommunications infrastructure and makes this region one of the most isolated in the world, which affects the quality of primary healthcare services. The primary healthcare system of the Napo River has 11 medical posts and 3 medical centers, and the hospital with medical specialists is the Regional Hospital of the city of Iquitos, located at the mouth of the Napo with the Amazon. Due to the transportation difficulties already mentioned, a patient's access to a doctor may require $10 \mathrm{~h}$ of travel, and even more if specialized care is required. The duration and cost of this displacement represents a serious barrier to the right to health of the Napo River populations and makes the public healthcare system less efficient, which must invest in displacement an important part of its limited resources. 
In order to strengthen the primary healthcare system in this area, the EHAS Foundation initiated, in 2008, a project that would offer telemedicine services to reduce the isolation of the health facilities in the Napo River. The objective was to put the health technicians of isolated communities in contact with their referring physicians through telephone and Internet services. Thanks to these services, health technicians can request help when diagnosing and treating patients, send epidemiological reports, share information about the inventory of medicines to avoid the loss of stock, coordinate emergency transfers, and send information about patients referred to the health center. But the deployment of a communication network in such a complex environment implies some challenges that cannot be addressed by a single organization while guaranteeing the sustainability of the network. That is why the EHAS Foundation made an identification of possible partners and formed a consortium to work on this project. The consortium consisted of 4 institutions: The EHAS Foundation (a non-governmental organization-NGO), Rey Juan Carlos University (URJC), the Pontifical Catholic University of Peru (PUCP) and the Regional Government of Loreto (GOREL). The following sections analyze the contribution of each of these institutions to the different dimensions of the sustainability of the Napo River network.

\subsection{Deployment of Telemedicine Services Stage}

This initiative was the first of its kind at a time when there were no precedents for networks of this type. From the technological point of view, it was necessary to investigate a low-cost solution that did not require connection to an electrical network (nonexistent in the Napo River), which could withstand the high temperatures and humidity of the forest along with storms and electric shocks while offering broadband connection for telephony and telemedicine services. The universities of the consortium were responsible for the research activities. URJC worked on adapting WiFi technology so that it could be used in long distance networks [2] and selected and configured the communication equipment of the project [14]. For its part, PUCP contributed its knowledge of the jungle environments, and was responsible for the adaptation of the systems to support the climatic conditions of the forest and to carry out the installation in the field. It should be noted that WiFi technology was already available in local markets at an affordable price, and technological sustainability in the short term was ensured when using it. Moreover, URJC transferred the research findings to the PUCP in order to guarantee the technological sustainability in the medium term. Additionally, the PUCP could use its role as a university to train technical staff in order to ensure technological sustainability in the long term.

On the other hand, the simple fact of having broadband services in healthcare centers was not going to contribute to improving patient care. For this reason, the EHAS Foundation, a Spanish NGO specialized in Information and Communication Technologies (ICT) and healthcare in isolated environments, was in charge of identifying the needs of the healthcare system, and based on that analysis, designed telemedicine solutions and appropriate care protocols for the Napo healthcare system $[15,16]$. These solutions were also transferred to the PUCP to facilitate the system's maintenance in the medium term.

\subsection{Economic Sustainability}

EHAS was responsible for coordinating the consortium and obtaining the non-refundable funds needed to deploy and maintain the network in the short term. This funding came from international development agencies such as the Madrid City Council (Spain) or the Spanish Agency for International Development Cooperation (AECID).

EHAS and PUCP worked together to develop a plan that would guarantee the economic sustainability of the network in the medium and long-term [6]. This plan identified the importance of involving the regional government of Loreto (GOREL) responsible for the public healthcare system in the region. The communication and telemedicine systems were expected to avoid unnecessary transfers of healthcare personnel and patients, and thus contribute to reducing fuel costs incurred 
by the GOREL. In return, the GOREL committed to allocating part of these savings to finance the maintenance of the telecommunications network in the medium and long term.

\subsection{Institutional Sustainability}

The telemedicine network has been operating since 2008 with a very high degree of acceptance by the health care staff and those responsible for the health system (GOREL). Health technicians feel more secure when being able to communicate by phone with their referring physician, which also increases the confidence of patients in the public system. However, GOREL has only partially exercised its commitment to maintain the network. The reason for this is that GOREL does not have personnel with adequate knowledge for the maintenance of telecommunications networks and the law does not allow it to allocate budget for the maintenance of this type of infrastructure. This situation forced PUCP and EHAS to continue exercising the responsibility of maintaining the network, but this situation was not sustainable in the medium and long term.

\subsection{Cultural Sustainability}

The PUCP was responsible for involving community leaders in order to provide cultural sustainability to the project. Thanks to this work, the Napo communities got to know and support the project: They provided spaces for the communication towers, contributed with the labor for its construction, supported the maintenance tasks, etc.

One of the difficulties during the first years was the increase of the traffic demand in the network due to the proliferation of applications like Skype, Facebook, or WhatsApp. The use of these services evidenced the need for communication of the healthcare personnel in these isolated communities. This was especially relevant among doctors and nurses, who, after receiving their degree, are usually assigned to work in rural communities for a year, under the condition that they will later be eligible for any other position within the public healthcare system. In fact, in recent years, personnel sent to the Napo River under these conditions have chosen this destination instead of others that are more accessible and less extreme but without access to the Internet. In order to prevent the use of social networks from collapsing the service, it was necessary to establish filters that would limit the use of these applications during working hours, but that would allow healthcare personnel to be in contact with their relatives and friends in their free time. In this sense, the cultural sustainability was dependent on the network being able to assume the users' demand for a greater bandwidth in the medium term.

\subsection{Political Sustainability}

The telecommunications sector usually has regulation designed to manage a scarce resource such as the radio spectrum, while ensuring that the service to end users offers an adequate quality. To deploy the Napo network, PUCP and URJC decided to use equipment that works in the ISM band (Industrial, Scientific and Medical), where it is not necessary to have a license to transmit telecommunications signals. Avoiding payment for these licenses greatly reduced deployment costs and contributed to ensuring the long-term economic and legal sustainability of the network. However, when working in these bands, the teams are susceptible to receiving interference from third parties that hinder or even impede communication. For this reason, this solution is appropriate only in rural environments where low population density means that there are few emissions in these frequency bands. This limitation prevents the scaling of this solution to urban or semi-urban environments but is adequate for the case of the Napo River in the short and medium term.

\subsection{Limitations}

During this first phase, the consortium managed to launch the initiative and guarantee its sustainability in all its dimensions in the short term, as summarized in Figure 2. However, GOREL's difficulties in assuming the economic and technical maintenance of the network became evident after 
the first years of work. In addition, from a cultural perspective, the inhabitants of this region share the communication needs of healthcare personnel and claim to be able to access telephony and Internet services. However, offering access to the entire population posed several challenges: The most used technology for this was mobile (GSM or 3G), but it requires licensed bands with high costs; it was necessary to design and fund a maintenance plan to prevent and repair service failures; and offering services to the entire population requires registration as a telecommunication operator and compliance with strict regulation.

In order to solve these limitations, it was decided to expand the consortium with the participation of mobile operators (MNOs) that would help to find innovative solutions. This resulted in a new phase of work that we describe in the next section.

\section{3G Deployment Stage}

Involving a MNO in the consortium could help extend telephony and broadband services to the entire population while generating revenues that would guarantee the economic and technical sustainability of the network in the medium and long term. However, the coverage of cellular services in Peru (and in many low and middle-income countries) does not usually reach rural communities with less than 400 inhabitants. Specifically, in Peru there are still 62,826 population centers without coverage as it is not profitable for operators to offer services in these communities using conventional deployment and business models. These models, exclusively based on satellite communications with high costs for backhauling, are not suitable for small communities offering reduced revenues that do not cover operating costs. However, it is difficult to design a solution without the operators because they have the licenses to operate in the cellular telephone bands, and they also have the knowledge and the necessary infrastructure to manage both the networks and the demand of the customers. For this reason, in 2013, the EHAS Foundation began to work on a new collaboration model that would allow the Napo network to be used to bring 3G telephony services (voice and data) to communities under 400 inhabitants.

It was a complex challenge that required reducing the costs of rural deployments through new technological solutions and proposing innovative business models that would exploit synergies with public administrations and other rural actors, such as NGOs. an additional difficulty was the scarce information available on traffic demand and the income that could be expected in these communities, making it difficult to design business models. To address these issues, the consortium was expanded and included a Mobile Network Operator (MNO), which was Telefónica, a manufacturer of 3G equipment (IP Access), a university expert in 3G systems (Polytechnic University of Catalonia-UPC), and the Investment Fund in Telecommunications of Peru (FITEL), which depends on the Ministry of Transport and Communications of Peru. This consortium obtained funding from the European Commission to investigate these issues in what was known as the TUCAN3G project [17].

\subsection{Technological Sustainability}

In this stage, UPC and URJC developed new technological solutions that allowed the use of wireless networks such as those of the Napo River to install 3G stations that would provide service to communities of less than 400 inhabitants. Likewise, IP Access provided 3G femtocells, which are base stations designed to provide $3 \mathrm{G}$ services to few users (as is the case in small communities) at a lower cost than conventional stations (designed for more users). The solutions proposed by URJC and UPC should be integrated by IP Access in their systems to ensure medium and long-term sustainability of the deployed technologies.

As a pilot, PUCP installed 3G femtocells in three Napo River communities and used the telecommunications network previously installed to connect these stations with Telefonica's central network in Lima, and thus provide service to Napo users. It should be noted that without the presence of EHAS and PUCP in this consortium it would have been very difficult to carry out a pilot deployment of these characteristics in such an inaccessible area. 
Although the incorporation of IP Access to the consortium was promising, eventually this manufacturer was not willing to adapt the equipment to a context as demanding as the Amazon rainforest, nor to other requirements derived from the deployment in rural communities. This restriction did not pose a serious problem for the TUCAN3G project, but it does reduce the potential for scaling and the long-term sustainability of the project. The reason is that, in subsequent projects, it has been necessary to use commercial equipment that is already adapted to the climatic conditions of the rainforest, but that involves a higher cost because it is not designed for small communities.

Satellite communications where still used as an external service for a shared connection between the Napo's terrestrial rural backbone and Telefonica's core network. Although the new MEO (Medium Earth Orbit) satellite constellation operated by O3B was considered, a GEO (Geostationary Earth Orbit) satellite was finally used because of the high costs of terrestrial stations for MEO constellations.

\subsection{Economic Sustainability}

EHAS and FITEL conducted a cost analysis of the TUCAN3G solution and proposed new business models based on the sharing of resources among different actors in the rural environments [12]. The results obtained from the pilot showed that the solution proposed by TUCAN3G was technically and economically viable in the medium term, since the reduction of costs achieved allowed for the maintenance of the network with the income of small communities [13]. However, these studies also showed that the income obtained was not sufficient to compensate the investment required in jungle environments, therefore it was still necessary to look for subsidies to scale this type of solution to other regions and ensure sustainability in the long term.

\subsection{Institutional Sustainability}

Telefonica played a key role in this consortium, since it allowed the use of its central network for the interconnection with other operators' networks and with the network of fixed telephony and Internet. In addition, Telefonica provided its experience in the operation of the mobile phone market, necessary to design an appropriate business model and ensure the operation of the network in the long term.

On the other hand, in this type of project, which ultimately seeks to favor local development, it is usually recommended to include public institutions that provide legitimacy, sustainability, and scalability to the initiative. In the TUCAN3G consortium, FITEL was included as the public institution responsible for the promotion of telecommunications in Peru. This incorporation gave the project access to information of interest to the Peruvian government, greater perspective of scaling, and greater facility to dialogue with the regulator and strengthen sustainability in the medium and long term.

\subsection{Cultural Sustainability}

As already mentioned, thanks to the work carried out by PUCP and EHAS within the framework of the first consortium, the Napo communities were receptive and even demanded 3G services. Moreover, having the service of a recognized operator in Peru (Telefonica) also contributed to the acceptance of the service among the population in the short, medium, and long term. In fact, some of the inhabitants of these communities already had mobile phones and telephone cards from this operator, which they used on their trips to the city of Iquitos. Likewise, the personnel transferred to the area to work in healthcare facilities, in educational centers, or in city halls already had their own mobile phones. However, the penetration of mobile telephony reached in this intervention was $27 \%$, which is far from the statistics offered by the ITU for developing regions ( $90 \%$ mobile telephony and $40 \%$ mobile broadband). This difference is explained by low-income communities, where there is usually a single cell phone for each family unit. If cell phone penetration does not grow over time, sustainability in the long term could be compromised. For this reason, it is interesting to complement 
the cellular telephone services with services to institutions, such as healthcare institutions, in order to diversify the income for the MNO.

\subsection{Political Sustainability}

FITEL's involvement showed the interest of the Peruvian government to support this type of initiative in the medium and long term. This interest is related to the responsibility of attending to the basic needs of the isolated populations, which cannot usually access their basic rights, and it is demonstrated that having communications is extremely useful to improve healthcare, education, and to promote the economic development of the community. For this reason, allocating public funds to subsidize deployments of $3 \mathrm{G}$ services or review regulatory frameworks is within the strategies of governments to improve living conditions in the most isolated communities.

Including Telefonica in the consortium allowed the use of licensed bands to offer the 3G service to end users, although the use of unlicensed bands was maintained for the backhaul network that connected the base stations with Telefonica's core network. However, when working with a commercial operator, it was necessary to consider the obligations that the regulator had regarding service downtime and repair times, which could result in sanctions and higher maintenance costs. This was a major concern for Telefonica throughout the TUCAN3G project, threatening the sustainability of this solution in the long term and which led the consortium to maintain conversations with the Peruvian regulator OSIPTEL. OSIPTEL considered that the quality of the service should be equivalent in both rural and urban areas in order to avoid maintaining the digital divide between these areas. For this reason, it was not willing to establish specific service conditions for rural areas, although this would entail an additional cost and therefore a barrier to the deployment of $3 \mathrm{G}$ services in these areas.

\subsection{Limitations}

Extending the consortium and involving international actors (companies, manufacturers, research centers, etc.) was a great opportunity to address the complexity of the problem and to identify new challenges, improving in this way the sustainability of the initiative, as shown in Figure 3. One of these new challenges was to establish mechanisms to scale up this type of solution. Scaling up TUCAN3G depended entirely on Telefonica, which on the other hand showed reluctance to replicate this type of solution due to regulatory restrictions and the required investment that was not covered by the expected income. In this context, both the regulator and the government have a key role because they have the responsibility to establish measures to encourage the extension of cellular coverage to isolated areas. The investment issue was covered by the participation of FITEL and by the investment strategies of the Peruvian government in the medium and long term. On the other hand, OSIPTEL was willing to establish a new role in the Peruvian legal framework to facilitate the deployment of rural 3G services: The Small Rural Operator (SRO). The SRO opened the door to avoid working with large operators such as Telefonica, and work instead with a small company, which is more flexible and better adapted to the characteristics of rural deployments, as discussed in the next section.

\section{Scaling Phase}

Once the technical and economic feasibility of the TUCAN3G proposal was verified, it was still necessary to verify its technical and economic viability in the long term. Large operators such as Telefonica are aware that they need to find solutions for rural environments due to pressure from governments and the need to attract new customers. However, rural communities have a high opportunity cost because they obtain more benefits in urban or peri-urban areas where competition is stronger. For this reason, Telefonica was reluctant to assume the responsibility of scaling up TUCAN3G and assuming the maintenance and management of this type of network. That is where the new role defined by the Peruvian regulation, the SRO, becomes important.

The SRO is an operator with license to deploy access and transport networks in communities without service, but does not have its own core network and therefore has to direct its traffic to the 
central network of a large operator in order to manage customers and interconnect with other networks. The MNO pays the SRO a rate for each minute of voice or per Mbyte of data transferred, and in return the SRO is authorized to use the frequencies of the MNO. Moreover, the SRO may require the regulator to mediate in the negotiation process if the MNO is not willing to reach an agreement. This opens the door to small operators interested in entering the market niche of rural communications.

From the MNO's point of view, the role of the SRO is interesting, since it allows them to attract new clients and meet the demands of governments while limiting the resources allocated to this goal. For this reason, the EHAS Foundation and some of its partners decided to start a new stage in which they included an SRO in the consortium. This SRO was Mayu Telecomunicaciones, which was the first SRO registered in Peru. The consortium was also expanded with PANGO, a Peruvian NGO that manages the Napo River healthcare system, and with Hispasat, a satellite operator that should connect isolated regions. In this way, the consortium was formed by EHAS, PUCP, Telefonica, Hispasat, and PANGO. The GOREL is also connected to this consortium since it is the owner of the communications towers and has the ultimate responsibility for healthcare in the region. The NAPO initiative is explained in the video "From TUCAN3G the NAPO-CAF project", whose link is available at supplementary materials.

\subsection{Technological and Economical Sustainability}

With this new consortium, EHAS has obtained the support of the American Development Bank (CAF), which is financing the deployment of $3 \mathrm{G}$ services in 15 communities in what has been called the NAPO Project. For CAF, this model was particularly interesting because it combines the interests of operators with the interests of developing, isolated rural areas. The involvement of a bank such as CAF strengthens the chances of scaling up this initiative, since it can offer loans to large operators, SROs, or governments that want to replicate this initiative.

In this new consortium, Mayu assumes responsibility for the maintenance of the telecommunications network. In this way, in exchange for being able to take advantage of the infrastructure already deployed in previous projects (and thus reduce their CAPEX and OPEX costs), Mayu offers connectivity for telemedicine services to EHAS, PUCP, and PANGO. This reduction in CAPEX and OPEX allows Mayu to assume the cost and maintenance tasks of the network, which helps to guarantee the sustainability and availability of the telemedicine services that operate over it.

As this is a very isolated region, the only possible terrestrial gateway to operators' core networks is in Iquitos city and it is a wireless backbone with no capacity available. Therefore, satellite communications are still needed to offer quality communications. Very powerful enterprises such as Google or SpaceX are working on LEO (Low Earth Orbit) satellite constellations that would be very interesting when available in the near future. Important organizations such as Geeks Without Frontiers, which share our interests and goals, are already taking positions in this sense. However, the best solution currently available is a GEO satellite. Hence, in the case of the Napo River, the deployed network connects through one of Hispasat's GEO satellite with Telefonica's core network, waiting for better terrestrial or spatial solutions to be available.

\subsection{Institutional Sustainability}

The incorporation of PANGO as manager of the healthcare system in Napo helps to guarantee that healthcare personnel really assume the telemedicine protocols and that remote support to healthcare technicians is foreseen by the organization. In addition, PANGO will work with EHAS and PUCP to include m-health solutions in the telemedicine network, which in previous EHAS research has been shown to reduce maternal mortality [18].

The role of Telefonica in the consortium would be dispensable once Mayu is incorporated, but this is explained by the interest of this multinational in expanding this model to other countries. Hispasat, in turn, seeks to study what role satellite communications can play in deployments in isolated areas which are note reachable with terrestrial links. 


\subsection{Cultural Sustainability}

For end users, the operator that provides the service is Telefonica, and therefore the SRO entrance does not modify the acceptability that the service had achieved with the TUCAN3G. This also contributes to scaling up and long-term sustainability, since the publicity and management of the client portfolio (billing, collection, claims, etc.) is still Telefonica's responsibility and the SRO does not have to allocate resources to those tasks.

\subsection{Political Sustainability}

In this stage, the goal was to formalize a model that was fully commercial and scalable, compared to previous stages with an experimental and research perspective. For this reason, those ultimately responsible for the service (Telefonica and Mayu) insisted on signing a detailed agreement that required several months of negotiations. The first step in the formation of the consortium was the agreement between Mayu and Telefonica. Although Mayu could request the mediation of the regulator (OSIPTEL), it was preferable for both parties to find a solution without intermediaries since it would be more stable in the medium and long term. Initially, the agreement between Mayu and Telefonica was restricted to voice services, but the consortium's proposal led to the extension of this agreement to include data services.

After guaranteeing the agreement between Telefonica and Mayu, a Memorandum of Understanding among all the consortium partners was formalized. This process was especially slow because Telefonica is an extremely large and complex operator, and different departments had opposite visions about this project. While the Rural Business Department saw it as an opportunity to provide coverage in the most remote communities, other departments considered the SRO model as a competitor that reduced Telefonica's profits. In addition, the delimitation of responsibilities was a crucial aspect for Telefonica's legal department, which had to approve the MoU. The internal discrepancies and the uncertainty associated with an innovative project made the negotiation process last more than a year. In this process, the management of the Innovation and Development area of Telefonica had a key role since they provided a more strategic vision with a greater perspective of internalization and the long term success of the initiative.

At the same time, EHAS and PUCP negotiated with the GOREL the assignment of the towers for this new stage, in which it was no longer just a matter of providing telemedicine services, but of offering voice and mobile data to the Napo communities. Being an initiative with a commercial aspect, GOREL had to review the agreement in more detail before proceeding to sign it, but the agreement was ready before the negotiation with Telefonica was completed.

\section{Conclusions}

Sustainability of communication services in rural areas is a complex issue that has been analyzed from different perspectives in recent years. This paper complements previous studies by considering the contribution of different partners to each dimension of sustainability. The experience here presented shows how alliances among different actors make telecommunications networks sustainable in isolated areas, but the scarcity of resources and the limitations of their isolation implies that scaling up these solutions depends on the funding of governments or international institutions. This funding would be justified by the contribution that communication services make in areas such as health, education, or economic development. But to guarantee this contribution, the role that NGOs and local universities play in managing the cultural and institutional aspects of the deployments is fundamental. On the other hand, the telecommunications sector is extremely complex, both from the technological perspective and from the point of view of managing legal and commercial aspects, which makes it almost indispensable to include operators. However, operators are often reluctant to adapt to the needs of isolated communities. The SRO can serve in the interlocution of these different perspectives by providing the specific knowledge of the sector combined with flexibility and adaptability. The balance 
inside these multidisciplinary alliances contributes to guaranteeing the sustainability of the initiatives in the long term, as shown in Figure 4.

It is also important to note that Mayu (the SRO) has been growing and expanding since it was created two years ago, and that confirms that there is an important market niche for this kind of company. Giants in the telecommunications sector such as Facebook and Google are also presenting their own ideas to connect a billion people who live in rural areas. Others such as OneWeb, Telesat and SpaceX are specifically working on manufacturing and deploying LEO satellite constellations. However, although these emerging technologies will certainly be of interest, pilots testing them and including a local development perspective with an integral sustainability approach will be needed. That is why we propose creating alliances that ensure that the deployment of telecommunication services in isolated areas brings a real improvement in living conditions in isolated regions.

Supplementary Materials: The following are available online at https://www.youtube.com/watch? $\mathrm{v}=$ PCcS3dqc3k4, Video title: "From TUCAN3G the NAPO-CAF project".

Author Contributions: I.P.-E. and J.S.-R. co-conceived the study. J.S.-R. proposed the methodology while I.P.-E. and A.M.-F. analyzed the information. The three authors contributed to the writing of the paper.

Funding: This work has been funded by the Development Bank of Latin America (CAF) and by the European Commission FP7 program through project TUCAN3G (IST-601102 STP).

Acknowledgments: The authors acknowledge the contribution of all partners of the NAPO consortium: EHAS, PUCP, Telefonica, HISPASAT, Mayu and PANGO.

Conflicts of Interest: The authors declare no conflict of interest. The founding sponsors had no role in the design of the study, in the collection, analysis, or interpretation of data, in the writing of the manuscript, nor in the decision to publish the results.

\section{References}

1. Saldana, J.; Arcia-Moret, A.; Sathiaseelan, A.; Braem, B.; Pietrosemoli, E.; Zennaro, M.; Simo-Reigadas, J.; Komnios, I.; Rey-Moreno, C. Alternative Networks: Toward Global Access to the Internet for All. IEEE Commun. Mag. 2017, 55, 187-193. [CrossRef]

2. Simó-Reigadas, J.; Martínez-Fernández, A.; Ramos-López, F.-J.; Seoane-Pascual, J. Modeling and Optimizing IEEE 802.11 DCF for Long-Distance Links. IEEE Trans. Mob. Comput. 2010, 9, 881-896. [CrossRef]

3. Rey-Moreno, C.; Bebea-Gonzalez, I.; Foche-Perez, I.; Quispe-Tacas, R.; Liñán-Benitez, L.; Simo-Reigadas, J. a telemedicine wifi network optimized for long distances in the amazonian jungle of Peru. In Proceedings of the 3rd Extreme Conference on Communication: The Amazon Expedition, Manaos, Brazil, 26-30 September 2011.

4. Nungu, A.; Knutsson, B.; Pehrson, B. On Building Sustainable Broadband Networks in Rural Areas. In Proceedings of the Technical Symposium at ITU Telecom World, Geneva, Switzerland, 24-27 October 2011; pp. 135-140.

5. Surana, S.; Patra, R.; Nedevschi, S.; Ben-David, Y.; Ramos, M.; Brewer, E.; Subramanian, L. Beyond Pilots: Keeping Rural Wireless Networks Alive. In Proceedings of the 5th USENIX Symposium on Networked Systems Design and Implementation, San Francisco, CA, USA, 16-18 April 2008; pp. 119-132.

6. Bebea-González, I.; Paco, J.; Linán, L.; Simó-Reigadas, J.; Martínez-Fernández, A. Management Framework for Sustainable e-Healthcare Provision. In Proceedings of the IADIS International Conference e-Society, Avila, Spain, 10-13 March 2011.

7. Pade, C.; Mallinson, B.; Sewry, D. an elaboration of critical success factors for rural ICT project sustainability in developing countries: Exploring the Dwesa case. J. Inform. Technol. Case Appl. 2008, 10, 32-55. [CrossRef]

8. Pade-Khene, C.; Mallinson, B.; Sewry, D. Sustainable rural ICT project management practice for developing countries: Investigating the Dwesa and Rumep projects. Inf. Technol. Dev. 2011, 17, 187-212. [CrossRef]

9. Rey-Moreno, C.; Sabiescu, A.G.; Siya, M.J.; Tucker, W.D. Local Ownership, Exercise of Ownership and Moving from Passive to Active Entitlement: a practice-led inquiry on a rural community network. J. Community Inform. 2015, 11.

10. Heimerl, K.; Hasan, S.; Ali, K.; Brewer, E.; Parikh, T. Local, sustainable, small-scale cellular networks. In Proceedings of the 6th International Conference on Information and Communications Technologies and Development, Cape Town, South Africa, 7-10 December 2013; pp. 2-12. 
11. Myers, E.N. Of Rhizomes and Radio: Networking Indigenous Community Media in Oaxaca, Mexico. Ph.D. Thesis, University of Oregon, Eugene, OR, USA, 2016.

12. Simo-Reigadas, J.; Municio, E.; Morgado, E.; Castro, E.M.; Martínez-Fernández, A.; Solórzano-Corral, L.; Prieto-Egido, I. Sharing low-cost wireless infrastructures with telecommunications operators to bring 3G services to rural communities. Comput. Netw. 2015, 93, 245-259. [CrossRef]

13. Martínez-Fernández, A.; Vidal, J.; Simo-Reigadas, J.; Prieto-Egido, I.; Agustín, A.; Paco, J.; Rendón, A. The TUCAN3G Project: Wireless Technologies for Isolated Rural Communities in Developing Countries Based on 3G Small Cell Deployments. IEEE Commun. Mag. 2016, 54, 36-43. [CrossRef]

14. Simó, F.J.; Martínez, A.; Osuna, P.; Lafuente, S.; Seoane, J. The design of a wireless solar powered router for rural environments isolated from health facilities. IEEE Wirel. Commun. 2008, 15, 24-30. [CrossRef]

15. Foche-Perez, I.; Ramírez, R.; Hirigoyen, G.; Balducci, F.; Simó, J.; Seoane, J.; Corral, J.; Martínez, A. An open real-time tele-stethoscopy system. Biomed. Eng. Online 2012, 11, 57. [CrossRef] [PubMed]

16. Prieto-Egido, I.; González-Escalada, A.; García-Giganto, V.; Martínez-Fernández, A. Design of New Procedures for Diagnosing Prevalent Diseases Using a Low-Cost Telemicroscopy System. Telemed. e-Health 2016, 22, 952-959. [CrossRef] [PubMed]

17. Website of the TUCAN3G Project. Available online: http://1locs.upc.edu/www-icttucan3g/ (accessed on 24 April 2018).

18. Martínez-Fernández, A.; Lobos-Medina, I.; Díaz-Molina, C.A.; Chen-Cruz, M.F.; Prieto-Egido, I. TulaSalud: an m-health system for maternal and infant mortality reduction in Guatemala. J. Telemed. Telecare 2015, 21, 283-291. [CrossRef] [PubMed]

(C) 2018 by the authors. Licensee MDPI, Basel, Switzerland. This article is an open access article distributed under the terms and conditions of the Creative Commons Attribution (CC BY) license (http:/ / creativecommons.org/licenses/by/4.0/). 